Delft University of Technology

\title{
Challenges in Advanced Solid Waste Separation
}

\author{
Bakker, Maarten C.M.
}

DOI

10.1142/9789813271050_0014

Publication date

2019

Document Version

Final published version

Published in

Critical Materials

\section{Citation (APA)}

Bakker, M. C. M. (2019). Challenges in Advanced Solid Waste Separation. In E. Offerman (Ed.), Critical Materials: Underlying Causes and Sustainable Mitigation Strategies (pp. 289-313). (World Scientific Series in Current Energy Issues; Vol. 5). World Scientific. https://doi.org/10.1142/9789813271050_0014

\section{Important note}

To cite this publication, please use the final published version (if applicable).

Please check the document version above.

\section{Copyright}

Other than for strictly personal use, it is not permitted to download, forward or distribute the text or part of it, without the consent of the author(s) and/or copyright holder(s), unless the work is under an open content license such as Creative Commons.

\section{Takedown policy}

Please contact us and provide details if you believe this document breaches copyrights.

We will remove access to the work immediately and investigate your claim. 
World Scientific Series in Current Energy Issues Volume 5

\author{
Critical Materials \\ Underlying Causes and Sustainable Mitigation Strategies
}




\section{Published by}

World Scientific Publishing Co. Pte. Ltd.

5 Toh Tuck Link, Singapore 596224

USA office: 27 Warren Street, Suite 401-402, Hackensack, NJ 07601

UK office: 57 Shelton Street, Covent Garden, London WC2H 9HE

\section{Library of Congress Cataloging-in-Publication Data}

Names: Offerman, S. Erik, editor.

Title: Critical materials : underlying causes and sustainable mitigation strategies /

S. Erik Offerman, Delft University of Technology, The Netherlands.

Description: New Jersey : World Scientific, [2018] | Series: World Scientific series in current energy issues; volume 5 | Includes bibliographical references and index.

Identifiers: LCCN 2018028408 | ISBN 9789813271043 (hardcover)

Subjects: LCSH: Raw materials--Research. | Strategic materials--Research. |

Mineral industries--Environmental aspects. | Sustainable engineering.

Classification: LCC TA404.2 .O43 2018 | DDC 333.8--dc23

LC record available at https://lccn.loc.gov/2018028408

\section{British Library Cataloguing-in-Publication Data}

A catalogue record for this book is available from the British Library.

Copyright (C) 2019 by Author

This is an Open Access ebook published by World Scientific Publishing Company and distributed under the terms of the Creative Commons Atribution (CC-BY) Licence.

For any available supplementary material, please visit

https://www.worldscientific.com/worldscibooks/10.1142/11007\#t=supp1

Typeset by Stallion Press

Email: enquiries@stallionpress.com

Printed in Singapore 


\title{
Chapter 14
}

\section{Challenges in Advanced Solid Waste Separation}

\author{
Maarten C.M. Bakker \\ Delft University of Technology, \\ Stevinweg 1, 2628CN Delft, The Netherlands \\ m.c.m.bakker@tudelft.nl
}

\begin{abstract}
Efficient mechanical and sensor-based separation of mixed solid waste into valuable secondary materials is a critical step in recycling and in the preservation of primary resources. To position the associated research field, we discuss backgrounds and links to societal and political developments within and outside the classical waste chain. An encompassing theory detailing separation processes is briefly introduced and explained using appealing examples. The roles of science and industry in the development of innovative technologies is clarified, finishing with examples of physical principles that are behind many contemporary separation technologies. Throughout, scientific challenges and technological advances are identified.
\end{abstract}

\subsection{Introduction}

Solid waste separation was traditionally the domain of a network of small businesses that relied mainly on manual labor and a means of transport, but this has developed into a modern waste management industry. The main drivers behind the changes were the increasing complexity and volumes of the waste, environmental legislation, and the sector's ambition to recycle more and better. Due to this impressive growth and matching societal impact, the academic world has recognized solid waste as an important field of science, technology and education. A transformation of our society into a circular economy model will widen the research scope. For example, the integration of product design, manufacturing and efficient recycling processes will help to close material loops and minimize material losses. Complementarily, the traditional business models of the recycling chain may have to be adapted or reinvented, as specialized recycling companies need

(C) 2019 The Author. This is an Open Access chapter published by World Scientific Publishing Company. It is distributed under the terms of the Creative Commons Attribution (CC-BY) License which permits use, distribution and reproduction in any medium, provided that the original work is properly cited. 
to collaborate more closely to create a viable supply chain of secondary raw materials (SRM) as a realistic alternative to virgin raw materials.

Separation is a key step in the recycling chain where mixed solid waste is converted back to reusable materials. Most of the solid waste is generated by sources such as building and demolition, industry, municipalities, agriculture, and automobiles. These waste streams may be subdivided into either source-specific or materials-specific streams, ${ }^{1}$ which then reveal in higher detail the origins and complex composition of the waste. As a principle, a separation technique aims to concentrate a target group of objects (i.e. pieces of waste) from the mixed waste, for which it utilizes their shared unique attributes. An attribute may be any physical or end-of-life (EOL) related product property of a piece of waste that can be utilized by a solids separation technique. In contrast, phase changing separation, as investigated in the chemical and metallurgical fields, requires the objects to be brought into a state of suspension or liquid melt before separation can be effected. The solids and phase changing separation methods may be complementary for end-of-life products with intrinsically mixed material compositions, such as waste from electrical and electronic equipment (WEEE), or alloys in metal scrap. In such cases solids separation can enhance the contents of the targeted materials or elements to a level where phase-changing separation can operate efficiently and attain the purity that is required by the market. Herein the focus is on solids separation, because the physics of the phase-changing techniques is quite different. This work aims to provide a view onto some of the major challenges in the field of solid waste separation. To this end, we give a brief overview of the field and typical research topics, propose a few possible scenarios in which the research connects to innovations and societal changes taking place in and outside the traditional waste chain, discuss in some detail the relation between waste composition and separation performance, discuss the mechanical and sensor based techniques, and show how seemingly simple physical principles can be utilized in effective separation techniques.

\subsubsection{Roots of separation science and technology}

In the 1970s public awareness and political mind-set converged on the idea that the protection of our environment was just as urgent as public health, ${ }^{2}$ which used to be the main motivation behind waste management. This resulted in the national and EU legislation ${ }^{3}$ that is aimed at protecting and preserving the air, water, land, and all biological life forms. The need for even greater protection boosted the development of technology and logistics 
of what is today a recognized waste management industry. In the EU, this industry drives the two main waste management options of recycling and waste-to-energy, as well as the least favored option of landfill. Especially in northern EU countries landfill is mainly reserved for hazardous types of wastes. The recycling option has gained preference due to increasing public awareness of the impact of materials production and waste generation on our society and living environment, and due to the political notion that many materials have a strategic value for the economy. Management options can also complement each other to provide a better waste solution. For example, non-recyclable residues from solid waste separation with high calorific contents may find their way to an incineration plant, while bottom ash residue from a waste incinerator contains a variety of metals that can be efficiently recovered using metal separators.

The state of the art in separation science and technology evolved naturally from fields like agriculture, chemistry and mining. But soon after waste management had evolved it was recognized that solid waste forms a class of materials on its own. After all, it consists of a wide variety of natural and man-made materials and comes in all shapes and sizes. To cope with such high complexity, the waste branch had to adapt the adopted technologies, thus sparking the evolution in separation technology that continuous to this day. In another response to the ever-increasing complexity of our waste, creative minds developed novel technologies based on previously unexplored physical principles that are unique to waste separation. For example, eddy current separators ${ }^{4}$ concentrate non-ferrous metals and near-infrared sensor sorters ${ }^{5}$ concentrate plastics according to material type.

\subsubsection{The circular economy}

Products in the linear economy were as a rule used only once and then discarded as waste in landfills and incinerators, while the concept of a circular economy does away with the notion of waste altogether. ${ }^{6}$ Instead, the lifespan of an EOL product should be continued indefinitely, preferably on the basis of its former functional value as in product reuse or refurbishment. If that is not an option, then on the basis of the intrinsic materials value as in recovery as SRM, which are the products of solids separation. To realize a circular economy, the present-day waste chains must be turned into united supply chains. This requires two major steps: the development of SRM-tailored quality standards and ensuring supply certainty. But even when these two hurdles are overcome and the circle is complete, the lifespan of SRM remains finite because no material or method of separation is 
perfect. Materials are subject to natural degradation, pollution, and wear, and to some degree will be mixed up with other wastes, causing them to finally end up as useless residue. This implies that the number of life cycles a material can sustain is finite and, moreover, that definitive solutions to this challenge must come from outside the traditional waste chain. We discuss four scenarios with a possible impact on solid waste separation. Each scenario could apply to a circular model that can support economic growth, innovations in product design and technology, and new business models.

Scenario 1: The producing industries may invest in reducing product complexity by making products easier to separate into pure materials by using fewer material combinations and by avoiding miniaturization involving material combinations. This improves the effectiveness of separation, increases SRM recovery rates and minimizes dilution of minor materials. In addition, sustainable material resources are needed to at least compensate for the loss of non-sustainable materials in the EOL phase.

Scenario 2: This scenario is based on the concept of leasing. The consumer pays for a product like a service, but the product remains the property of the producer. When buying a new product the consumer turns in the old one. The advantage is that the producer becomes the problem owner and the party responsible for the collection of retail-returns. This reduces the amount and variability of the wastes cycling through the public waste chain, provided the responsible producer also organizes the reuse, refurbishing, or recycling of the EOL.

Scenario 3: In a deposit scheme such as for PET bottles or glass beer bottles in the Netherlands the EOL are returned to the producer through retail outlets. In principle this is a prime example of circular reuse. However, the variety in consumer products is practically infinite so this scheme cannot possibly be extended to all EOL. But in a realizable future a product could be provided with a unique tag such as a bar code that reveals for example the manufacturer and the materials composition. Such a tag could also serve for price and storage information as is already common practice in retail. The main issue is that fully automated sensors should be able to read this tag in the EOL phase, for example at the recycling plant. Thanks to the tag, valuable dedicated materials can be returned to the original producer in a way similar to the deposit scheme. If the materials hold no special value for the original manufacturer, the tag reveals materials information that can facilitate efficient recycling by another route. 
Scenario 4: As we have entered the information age, it is expected that Information and Communication Technology (ICT) will also find its way into the secondary supply chains of the circular economy. The value added by information and data in the 'upper world' of production, consumption, and services offers a glimpse of what may lie in store once comparable ICT infrastructure is in place in the secondary supply chains with what used to be waste. The chains can provide information and quantitative data through sensor systems on the origins, volumes, and composition of the waste, the efficiency of collection and separation processes, and on the composition, quality, and available volumes of the produced SRM. It is expected that this information can generate a trading and commercial value independent of what is generated at the whimsical secondary materials markets.

\subsubsection{Sensor sorting and quality inspection}

During the last thirty years, sensor technology in waste separation was boosted by the rapid development of advanced sensors, in particular the types that allow moving objects to be scanned without making physical contact. Though sensors were around for much longer, it wasn't until the technology reached a mature level of performance and price that it became more widely adapted in the waste industry. In an application, the waste objects are deposited on a conveyor on which they should be in rest before reaching the sensor. The sensor scans each object individually after which software interprets the sensor data to decide in real-time on the presence of the targeted unique attributes. If detected, a signal is sent to an actuator that mechanically moves the object to a separate stream. The price of a sensor system tends not to be determined by the physical sensor, but rather by the actuator system and the electronic hardware required to process all the acquired data in real-time. A wide variety of sensors is available to detect all kinds of object attributes, be it geometrical (e.g. length, height), physical (e.g. magnetic or chemical properties), or related to the functional product origins (e.g. color, shape, material build-up).

Actuators also come in a wide variety of types, where air jets and systems for mechanical pushing or hitting the object are favorites. A major research challenge that could mean a real breakthrough in sensor sorting is the development of a new generation of compact, low-energy actuators that can be stacked along the length of a conveyor belt. This would enable the extraction of multiple products with different desirable properties in one processing step. Key in this development would be that all actuators 
are activated through one and the same sensor unit to limit the costs. In principle, the possible number of stacked actuators need only be limited by the length of the conveyor. This development would result in a considerable cost saving for sorting, because the present generation of sensor sorters can only make two or perhaps three products in one step.

A distinctive advantage of combining different sensors in one system is that it can identify different useful product properties in one step. For example, in the case of sorting waste plastics one could discern the polymer type, color, and presence of polymer additives at the same time to sort a tailor-made product for the end user.

Since a sensor scans each individual waste object, the separation of large objects is more efficient than small objects, because throughput capacity is typically measured as a mass rate. When feeding a sensor sorter the objects should form either a monolayer on a conveyor ( $2 \mathrm{D}$ sensors) or be put in a moving single file of interspaced objects (fixed point sensor). It is a challenge to achieve the highest feeding rate that the sensor can sustain without making too many errors, which requires the densest possible homogeneous distribution of objects without overlapping or touching.

Sensors can also be employed without an actuator to inspect the composition of a batch of SRM, which operation is called quality inspection. In this case, the feed rate of the sensor may be higher than its maximum capacity if the objective is to sample the waste stream. By scanning a representative number of samples per time interval, the sampling statistics return an average composition per time interval with associated uncertainty. The uncertainty may be reduced by increasing the time interval or by increasing the sampling rate, whichever is cheaper or more practical.

\subsubsection{Waste collection systems}

Waste was traditionally discarded through an efficient scheme of compacting collection trucks and disposed of in landfills or incineration plants. This strengthened the public belief that waste has no value and can easily be made to 'disappear'. This applied to all waste streams with perhaps the exception of the most hazardous for which more strict regulations were in place. Here, we focus on household waste because we all experience this type of waste first hand. As recycling gained momentum as the preferred waste management option, a rethinking was required of the traditional mixed waste collection scheme. Separate collection schemes were introduced for all kinds of recyclables to minimize the contamination, material mixing and volume per type of waste stream. In combination with automated 
separation technologies these schemes improve the material recovery rates (i.e. the percentage of materials successfully separated) and lower the costs for the recycling plant. In turn, better separation improves the achievable recycling rates (i.e. the percentage of collected materials actually reused in new applications). Modern waste collection is no longer just a logistical operation as it should also motivate people to separate their waste in the easiest and best way possible. In turn, the main actors in the waste chain have the obligation to be transparent about the achieved recycling rates and inform people about their personal waste footprint. It is here that synergy can be created by combining efficient automated separation technology at the recycling plant with people's personal involvement in separate waste collection and applying a system of feedback to help people to reduce their waste footprint.

A separate collection scheme is aimed at isolating and safeguarding the valuable recyclables by using a system of separate bins or otherwise distinguishable collection means such as colored bags. This ensures they can be processed effectively in the recycling plant, provided people throw the right waste into the right bin. The waste pickup frequency will vary depending on the volume and nature of the recyclable. For example, smelly biogenic kitchen waste has to be picked up more often for hygienic reasons. Other recyclables have a quite low bulk density and appear in high volumes, which make their separate collection costly as more truck rides are required to collect the same total mass. Examples are packaging plastics and the more season-dependent garden waste.

For separate collection different options are available. People may separate and collect inside their home, or nearby in underground containers, or in a farther removed recycling street in case of more coarse types of waste. An example of a more recent approach of home-separation is the use of colored plastic bags. All these bags may be deposited in the same collection bin or underground container, but the recyclables remain isolated. Moreover, only one pickup moment is required for all the bags, which lowers the costs of collection. The collected bags are automatically separated at the recycling plant using sensor technologies. To limit the number of color bags needed in the home, different recyclables could be combined in one bag provided they can be efficiently separated at the plant using mechanical separators. For example, plastics, metals and drinking cardboards (called PMD fraction) form a large part of the daily household waste volume and are collected together in several EU countries. However, it is not yet clear if the recovery and quality of the plastics from PMD are comparable to what is possible using separate plastics collection. 
As an extension to the colored bags system, it is technically possible to tag bags before they are distributed to the local population with an information carrier that can be read by automated sensor systems at the recycling plant. For example, a bar code may reveal the area and street of the pickup location. Other sensors can perform a quality inspection of bag contents and build a database with EOL information. This can be coupled to the area tag to reveal the efficiency of home separation and waste volume and consumption pattern per waste pickup location. This information can be used to steer the recycling chain, make local improvements to waste collection and inform people locally of their average waste foot print.

The option to separate only in bins outside the house can be a workable alternative in low-rise and open urban areas with sufficient outdoor spaces. But the success of any separate collection system depends on people's active involvement, and that depends on quite a few factors such as walking distance to the containers, ${ }^{7}$ available space for temporary waste storage, effort and convenience, aesthetics, and waste pickup frequency in a trade-off with collection costs. Many different collection systems are employed and tried in the Netherlands. Some of these are combined with positive or negative financial incentives, such as differentiated waste taxes (called Diftar) and penalties for putting out waste at not approved locations or at the wrong time. There are a few distinct successes with separate collection and recycling in the Netherlands, for example packaging glass, paper and cardboard waste and metal scrap. However, there still is a major problem with packaging plastics as the costs of separate collection prove too high in combination with disappointing recycling rates. This indicates that, even many years after its first introduction, separate collection is still in a transition phase and warrants a higher level of academic involvement to understand the bottlenecks and to develop efficient solutions. Especially the connection between technology, social-cultural influences and human behavior is a crucial cross-road of academic studies that in collaboration can help the separate collection concept to become a successful system of the circular economy. $^{8}$

Instead of asking people to sort their waste, one may also use automated technology to sort mixed waste at the recycling plant. This concept continues the mixed waste collection system, though shredding and high compaction in the waste truck can no longer be allowed for obvious reasons. Advantages of this system are the low cost of collection and it is convenient for big cities with old and crowded centers that cannot easily accommodate more waste bins and underground containers. The technology needed for 
mixed waste separation can be classical and readily available, which makes this system also less of a risk where it concerns return on investment. But because it also does not require any active collaboration from the people, it probably does not change people's perception of waste very much or their attitude towards reducing their waste footprint. The technical disadvantages are more cross-contamination and material mixing, which lowers the potential recycling rates in comparison to a well-implemented separate collection system. As such, mixed waste separation may be regarded as an evolutionary technological solution for the big cities and a temporary step towards advanced separate collection.

Lastly, manual separation cannot be ignored as a factor in modern waste separation. This human-based activity is still a necessary step in the separation of the largest solid waste streams, and not only in low-wage countries. Manual labor is, for example, deployed in the disassembly of electrical and electronic appliances which consist of a few large homogenous parts (e.g. casings) and smaller complex parts (e.g. wires, electronic circuit boards and power supplies, electric motors). Handpicking is a longstanding contribution of manual separation where one or more persons pick up and remove specified objects from a conveyor belt. The minimum object size a person can routinely pick up is about $25 \mathrm{~mm}$, but bigger objects make for more efficient handpicking as they can be picked at a rate of up to 20-30 per minute. Manual labor in separation lines may seem to contradict the advancement of technological waste management, but a plausible motivation is the uncertainty about how long complex EOL will keep on evolving and diversifying. After all, electronics such as smartphones and smart household devices with sensors keep on changing and diversifying every year. As a consequence, recyclers postpone substantial investments in innovative technologies due to uncertainty if they will be able to cope with the continuous increase of EOL complexity. Instead, they rather rely on manual labor to break down complex EOL to waste streams that can be dealt with by proven technologies.

\subsection{Theory of Solid Waste Separation}

The main condition for separation of a group of targeted materials (objects) from a mixed waste stream is that the group must share at least one attribute that sets them apart from all the other waste objects. Based on this attribute, a separator could ideally isolate all the targeted materials. Unfortunately, separators can make two possible errors. A targeted 
object that is not separated into the main product is called a false negative, and a non-targeted object that is unintentionally separated into the main product is called a false positive. Though this terminology is mostly used in connection to sensor-based sorting, the concepts can be consistently extended to mechanical separation techniques. The cause of these errors is either: the choice of attribute utilized for separation turns out to be not that unique and is also shared by non-targeted objects (false positives); or it is not shared by all the targeted objects (false negatives); or the separation process is not selective enough, which may lead to either error. We discuss these a bit further.

The unique attribute and corresponding separator are chosen by the plant operator on the basis of the value of materials and by sampling the contents of the waste batch. However, for any waste batch there is a certain probability that non-targeted materials share that attribute. In addition, separation technology is effective only within certain physical ranges of the attribute by which some targeted objects may be missed. For example the eddy current separator (ECS). Its effectiveness in accelerating a nonferrous metal object through electromagnetically induced currents decreases rapidly with size and electrical conductivity. On the other hand, it can also not separate too large and heavy metal plates. In general, the probability of an error may be reduced by utilizing more distinguishing attributes in the separation. Unfortunately, mechanical separators are usually specialized and aimed at one particular attribute. Therefore, if effective separation requires more attributes, different separators need to be put in series and that translates into higher processing costs. In contrast, sensor units can be easily extended with other sensors and sorting can be done on the basis of multiple distinguishing attributes in one processing step.

Feeding a continuous stream of solid objects into a separation process invites dynamic object-object and object-machine interactions. For most part these result in the intended deterministic separation. However, the dynamics of a large stream of solid particles allows for more complicated dynamics that can negatively influence the result. We name here: segregation (clustering of objects with similar attributes); agglomeration (different objects connecting/adhering and then behaving as one composed object); incidental random occurrences (objects behaving in an unpredictable fashion). If the latter random behavior results in a separation error it is referred to as a dropout. Another undesired behavior of solid objects is entrainment, where objects are locked in a stream and forcecarried into the wrong product. For example when a light object is enclosed 
by heavy ones and forced to follow them into the heavy product. It is noted that entrainment can also have a positive connotation if the mechanism is utilized to extract or concentrate materials. Segregation can negatively affect the separation when it results in the formation of object clusters inside the machine that restrict the proper flow and intended motions of the targeted objects. Agglomeration negatively affects the separation when a targeted object connects to a non-targeted object. It is important to use the correct feeder that minimizes segregation and frees objects before the separation action takes place. This is usually a shaking feeder that evenly and homogenously distributes the materials into the machine. If agglomeration is a problem the responsible mechanism has to be determined. For example, moist materials may have to be dried first to free the individual particles. On average, the influences of segregation, agglomeration and entrainment on the separated products are fairly repeatable mechanisms for a specific combination of feed materials and separation process. As such, their average contribution to false positives and false negatives can be determined experimentally by processing a prepared test batch.

Dropouts are of a quite different nature as they occur incidentally and unpredictably. As such, their occurrence may be modelled using a fitting probability function, perhaps a Poisson distribution. An experimental test may also give insight into this type of error by examining the standard deviation of the detected false positives and negatives. Mechanical separators are always fed in bulk, which invites many possible interactions between the objects and the machine. The probability of dropouts may therefore be influenced by the feeding method and feed rate. But sensor sorters require a more controlled form of feeding, which limits the possible interactions and reduces the probability of dropouts.

\subsubsection{Attribute space and effective separation}

The performance of a solids separator can be mathematically described using separation system theory. This theory does not describe the physics, but rather the effective behavior of the machine for a specified waste batch and the resulting composition of the products. It can incorporate a continued separation line, binary and multiple separations in cases where a machine creates more than two products, and, though this seldom happens, a line where a part of the processed material is looped back to an earlier point in the separation line. We briefly introduce the basic concepts behind this theory. 
First, all individual objects in the waste batch are documented according to a list of physical, geometrical and EOL-related attributes that may be relevant to their separation. Each physical or geometrical attribute is quantified by a parameter (e.g. length, density, RGB color values, magnetization). Among all the objects in the waste batch the parameter varies within a finite range of values. An EOL attribute (e.g. material type, color, description of shape) is specified by an identifier. The possibilities for the identifier must be arranged in a fixed order. For example, for a certain waste batch the attribute 'material type' may have three identifiers, ordered as 'plastic', 'metal' and 'wood'.

Next, each object is assigned to a material class, where each class forms a different marketable product. The exception is the residue that contains the objects that do not fit in any other material class. In practice, classes are chosen with a view towards the potential market price and the available separation techniques. A class may also be related to only EOL attributes. For example, the class 'plastic bottles' combines identifiers from the attributes 'material type' and 'description of shape'. The main issue for successful separation of all the objects in a material class is that they must share unique attributes that set them apart from all the other classes. Nevertheless, depending on the order of separation, different classes may have overlapping attributes without negative consequences for separation. For example, when separating the classes 'plastic' and 'plastic bottles', one should first separate the plastic bottles as they are more uniquely defined than 'plastic'.

Each listed attribute is assigned an axis in a multi-dimensional space, which we call the attributes space. To simplify the interpretation, the parameters are discretized using arbitrary axes (i.e. in the sense of nonlinear axes), so as to span the multi-dimensional attribute space with simple shaped elements. This includes axes assigned to EOL attributes which are occupied by ordered identifiers.

To each element in attribute space the number of objects that comply with that specific set of attributes is assigned. Figure 14.1 shows a simplified example for different types of aluminum scraps. The distinguishing attributes are 'size', 'density' and 'conductivity', which span a 3D attribute space. For visualization we showed the projections onto two cross sections with 'size' as common axis.

An ideal separation technique is sensitive (as in effective) only to the unique attributes of the targeted material class. However, in reality a separation technique is also to some extent sensitive to other attributes. The 

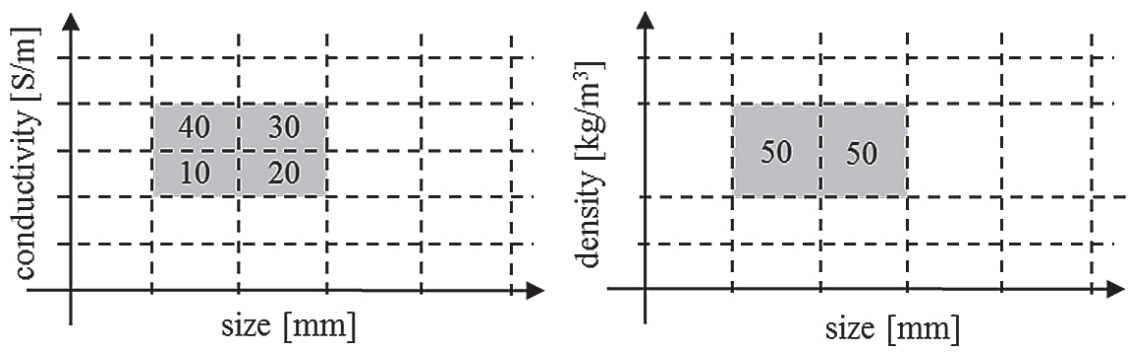

Fig. 14.1. Example of a 3D attribute space. Indicated are projections on two cross sections of this space, harboring 100 different aluminum scraps.

sensitivity spans a subspace in attributes space which we call the separation subspace. Note that, as a subspace, it conforms to the discretization of the attributes space. Each element in the separation subspace is assigned a sensitivity on the scale $0-1$, where the complement to 1 defines the reduced success to separate an object with those specific attributes. For elements where the separation subspace coincides with the targeted unique attributes, the complement to 1 defines the chance of a false negative. For elements where the separation subspace does not coincide with targeted attributes, the sensitivity itself defines the chance of a false positive. As such, false negatives are due to a lack of sensitivity and false positives arise because the sensitivity extends to parts of attribute space that are occupied by non-targeted attributes from (most likely) non-targeted objects. It is noted that statistical spread in the sensitivity can readily be accounted for by introduction of probability distributions.

Figure 14.2 shows two examples of discretized 2D separation subspaces where the sensitivity is indicated in grey-scale. Figure 14.2(a) represents an eddy current separator (ECS), which is sensitive to non-ferrous metals. The sensitivity for metal scraps decreases with size and electrical conductivity, while too large and heavy scraps can also not be separated. This contributes to the false negatives. Because a high conductivity is mainly associated with metals, the chance of false positives in an ECS is mainly due to agglomerated objects that include a small piece of metal (e.g. a metal screw in a large piece of wood) and dropouts.

Figure 14.2(b) represents a float-sink tank where the float fraction is considered the main product. Materials that are generally separated well are much heavier or much lighter than the cut density and have a reasonable size. Small, heavy particles may still float due to frothing, adherent air bubbles, air inclusions, or agglomeration with light materials, in which case 


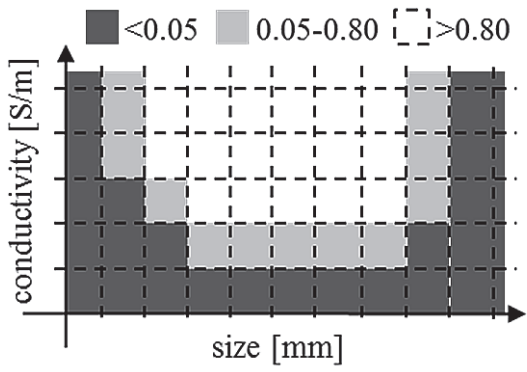

(a)

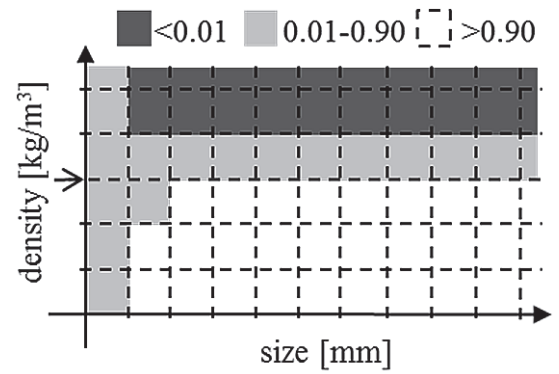

(b)

Fig. 14.2. Examples of 2D separation subspaces. The sensitivity is shown in grey scale. (a) Eddy current separator. (b) Float-Sink tank, where the arrow indicates the cut density.

they contribute to the false positives. Materials with a density a bit higher than the cut-density sink slowly and have a tendency to be dragged by the flowing liquid into the product bin, in which case they also contribute to the false positives. Small particles lighter than the cut density may still sink due to agglomeration with heavy materials, which contribute to the false negatives.

Now that the basic concepts of separation system theory are explained we can give a precise definition of the effective separation technique. One selects the technique for which high sensitivity covers the part of attributes space occupied by the unique attributes of the targeted material class, while outside that part of space the sensitivity decreases rapidly to zero. If this type of separator is not available, an alternative is to first optimize the attributes space of the waste batch to fit the separation subspace of the available technique. This can be done by applying a precursor process (pre-process for short). There are two types of pre-processes: one that leaves the existing object attributes intact and one that changes them. The first is typically a separation process that removes the fraction of targeted objects from the batch whose attributes fall outside the separation subspace of the available technique. For example, a sieve can remove all the small objects from the batch in case the sensitivity of the available technique for the attribute 'size' is restricted to large objects. Moreover, sieving to size reduces the material volume that has to be processed by the available machine.

The second type of pre-process may be comminution (e.g. breaking, milling, rolling), liberation (e.g. shredding, hammering), or surface 


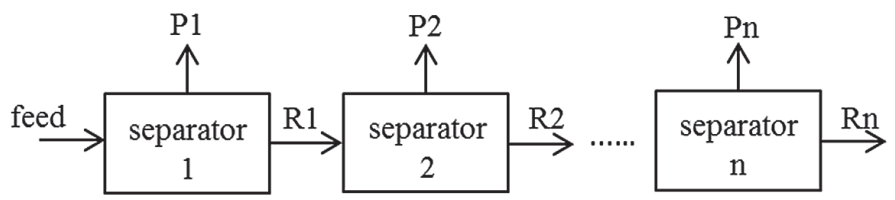

Fig. 14.3. A line of connected binary separators.

treatment (e.g. grinding, acid attack, spallation). Comminution aims to break objects down to prescribed size, liberation aims to break up heterogeneous objects into as big as possible homogenous objects, and surface treatment aims to modify surface properties like roughness, color or coating. These processes can change attributes as well as create new objects and new attributes, and therefore demand a new documentation and classification of the processed waste batch. A major research challenge is to detail the attributes space created by such a process on the basis of a transfer function and the known attributes space of the feed.

A series of different separators can turn a batch of mixed waste into a number of valuable products. Designing a separation line starts with knowledge of the material classes and their discerning attributes. From here on the challenge is to determine the products that can be concentrated successfully using the available techniques.

Figure 14.3 shows a generic line of binary separators that is continued on the non-concentrated fraction, which is here referred to as residue (labels $R$ ). The concentrated fraction is a product (labels $P$ ), while the other objects end up in a residue. It is noted that it is also possible to continue the line along a concentrated fraction if further separation is required. The separation line invites a bit of thinking about false positives and negatives. For example, if an object is erroneously assigned to the product of the first separator it is a false positive in that product. However, it is removed from the feed down the line and will never end up in the correct product, which we classify as a false negative for the correct product. Vice versa, if a targeted object is erroneously not separated in the first product, it can only cause a false positive down the line, except if it ends up in the final residue. What this example shows is that the definitions of false positive and negative are also consistent for separation lines, but that their cause may lie with another separator. Ergo, the ordering of the separators plays a role and it is efficient to set the most effective separator up front to reduce the chance of false positives down the line. In another strategy, it may also be efficient to put up front the separator that takes out a large material 
volume. This reduces the feed rate and capacity required of the machines down the line.

\subsubsection{Engineering considerations}

The throughput capacity of an industrial separator is limited in terms of volume and mass rate, which sets demands to how and how much material can be fed. Feeding can be done from a bunker or shaking feeder to bring objects in free fall into a machine or to deposit them on a conveyor belt and cover it with either a monolayer or a thick layer of materials. The feeding method serves to provide continuity to the separation process up to the maximum throughput capacity, and to render the separation effective within the required separation subspace. Machines come with variable settings that serve to facilitate limited variations to the separation subspace to gain a better match with the targeted part of attributes space. On the other hand, during prolonged operation the machine may be subject to wear, tear, and caking due to the continuous feeding of large objects and fine materials. These maintenance-dependent factors may change the separation subspace during operation and negatively affect the performance of the machine. The moisture content of waste can be a major point of concern. Water may adhere to objects, invade porous objects by the capillary effect, or loosely adhere as free water between objects by water bridges. The impacts of moisture are added mass and agglomeration of mostly small objects and fine materials (e.g. mineral grains), which adhere to moisture more effectively due to their relatively larger surface to volume ratio. In these ways, moisture can modify the attributes space spanned by the objects in the waste batch. On a similar note, adhered moisture on machine parts may affect the separation subspace of the machine due to stick or slip or due to caking of a multitude of fine materials.

Full separation of mixed waste involves several pre-processing and separation steps. To arrive at an all-encompassing theory for these connected processes the object attributes must be documented and the sensitivity of each machine must be determined. The practically infinite number of possible material/elemental combinations of complete and shredded EOL objects can only be addressed by the introduction of a system of materials classification, such as was introduced in the preceding section. To define classes one may take a pragmatic approach that is consistent with accepted recycling practices: a material class forms a saleable SRM product that may be composed of either pure materials or of a specified mix of EOL parts and homogenous materials. Within this definition, a material class may still 
require further solids separation and/or phase changing separation before it is pure enough to be acceptable for reuse. A few examples of mixed SRM are 'plastics', 'metal scrap', 'paper and cardboard', and 'printed circuit boards'. The criterion for selection of an object from a mixed waste batch in a material class is the question if the material variety brought in by that type of object and the combined mass of all the similar objects in the batch could negatively affect the quality or salability of the class. This implies that a small amount of 'material pollution' in the class is acceptable as long as one gains the targeted material, but one cannot accept a certain polluting object in the class if there are too many of those. If that is the case, they should be put in a class of their own. On a critical note, this selection criterion allows for dilution and associated loss of foreign elements and materials when their total mass contribution to the class is deemed negligible. However, it is consistent with the state of the art: it is unfortunately not economically feasible to separate with a resolution that recovers all minor materials in mixed solid waste.

\subsection{Separation Techniques}

The mechanical and sensor-based separation techniques are the enablers of modern solid waste separation. Both types are introduced and discussed in terms of potential and limitations. We also point out the design steps in which scientific research can join forces with industry to transform a promising principle for mechanical separation into a proven technology. Finally, we introduce a few successful principles that formed the basis for many contemporary mechanical separation technologies.

\subsubsection{Advanced data processing in sensor sorting}

The separation subspace of a sensor sorter is mainly determined by the sensor unit, consisting of the physical sensor and a stimulus. The stimulus excites the waste objects (e.g. optimized light source, x-rays, electrons, magnetic field) and the sensor detects their response. The response should be selectively sensitive for the unique attributes of the targeted objects. The raw sensor data is transferred to a real-time processing unit that contains software for data interpretation and decision making. The sensor system is complemented at the front end with an adequate system to present the objects to the sensor unit (e.g. a shaking feeder and conveyor belt) and at the back end with a mechanical actuator that removes the identified objects from the waste stream. The actuator should meet the resolution of 
the sensor unit and the speed of the data processing unit. The design of the software depends on how one can extract the information from the raw sensor data. for which there are roughly four strategies.

1. The data provide direct evidence of the unique attributes, e.g. if the detected signal exceeds an uncertainty level.

2. The data allow the unique attributes to be quantified. There are two different approaches. The first is calibration, where the detected signal can be compared to a documented reference, such as a lookup table or a formula. This has the advantage that it does not require knowledge of the physics behind the generated signals. The disadvantage is that the reference requires calibration samples to be prepared, analyzed and documented. The second technique is quantitative measurement, which requires some processing to translate raw data to quantitative attribute values. This has the advantage that one can utilize signal and selectivity enhancing measurement strategies such as compensating, modulating, and differential measurement techniques.

3. The data carry indirect quantitative information on the unique attributes due to their influence on a specified physical process, by which the attributes may be detected and quantified through their level of influence.

4. The data carry merely coherent information on the unique attributes due to their influence on several physical processes, but these influences are not well understood. Nonetheless, using statistical techniques the coherent influences of the attributes may be detected and classified with statistical certainty.

Option 1 forms the basis for a cost-efficient technique as it puts minimal demands on data quality and real-time hardware. In option 2 the calibration techniques share similar benefits, but have the major drawback of requiring extensive sampling and analyses of the targeted materials and attributes to adapt the lookup table every time these waste objects change significantly. The quantitative measurement techniques in option 2 set higher demands on data quality for a reliable translation from raw sensor data to a physical interpretation. Option 3 may also be called model-based measurement. There, a physical model is constructed that incorporates the influence of the targeted attributes to predict the collected sensor data with required accuracy. ${ }^{9}$ By inverting the model equations the attributes can be quantified on the basis of the collected sensor data. In option 3, data quantity and quality play important roles. The main challenges are to suppress numerical 
sensitivity for small data variations (e.g. sensor noise) and to make sure the reconstructed attribute parameters form a unique solution. Both challenges are inherent to data inversion problems. ${ }^{10}$ Option 4 allows for a range of statistical techniques to be applied. The mathematics behind the algorithms is generally aimed at isolating coherent features in the data and projecting them onto a virtual space in which objects with detected shared attributes are clustered. ${ }^{11}$ Sorting of the clustered objects is effective only if these shared attributes are 'unique enough' so as to avoid different object clusters from overlapping. ${ }^{12}$

\subsubsection{Statistics of materials feeding}

Statistics are an important tool to help understand the feeding constraints of mechanical and sensor-based separators, because there is no mechanical system that is able to control the motions of each individual object when feeding them in large numbers. Here we focus on the main aspects of monolayer feeding of a sensor, where the maximum feed rate depends on how densely the conveyor belt can be covered with objects without too many overlaps. A 2D sensor such as an infrared or visual light camera cannot always reliably distinguish overlapping or even touching objects, so some spacing is desired. A typical feed system involves a shaker that provides a uniform feeding along the width of the belt. To assure a uniform feeding in the length direction the belt must move faster than the objects falling from the shaker. The object distribution on the belt follows a probability distribution which we reconstruct using a Monte Carlo simulation. For simplicity, all objects are assumed flat and square with length $d$, and all objects fall perfectly aligned in a row along the width of the belt. The maximum feed rate for ideal $100 \%$ belt coverage for this case is $f_{M}=L V / E(d)^{2}$ objects/s, where $\mathrm{L}$ is the width of the belt and $\mathrm{V}$ the belt speed. The function $E(d)$ is the statistical expected value of the length of the objects plus a required minimum spacing of $2 \mathrm{~mm}$ between any two objects. The feed rate is a fraction $f$ of the maximum feed rate $f_{M}$. The parameters used in the three simulated cases in Fig. 14.4 are shown in Table 14.1. Case 1 uses only $5 \mathrm{~cm}$ objects and cases 2 and 3 use objects in the range 2.5-10 cm with an equal probability for each size. Figure 14.3(b) shows the function $A_{O} f f_{M} /\left(\mathrm{LvN}_{O}\right)$, which is the percentage of the belt surface that could be perfectly covered with objects without any overlap. $N_{O}$ is the total number of released objects and $A_{O}$ is the total surface of the objects on the belt. 


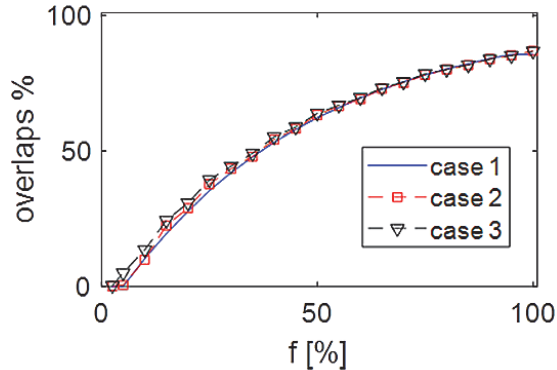

(a)

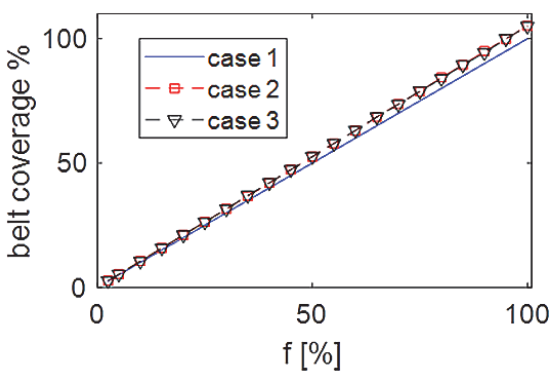

(b)

Fig. 14.4. (a) Percentage of overlapping square objects as a function of fractional feed rate $f$ : all $5 \mathrm{~cm}$ (case 1) or size range 2.5-10 cm (cases 2 and 3) (b) Average belt coverage.

Table 14.1: Parameters in Three Simulations of 2D Monolayer Belt Feeding and Object Overlap.

\begin{tabular}{lccccc}
\hline Case & $\mathrm{d}[\mathrm{cm}]$ & $\begin{array}{c}\mathrm{E}(\mathrm{d}) \\
{[\mathrm{cm}]}\end{array}$ & $\begin{array}{c}\text { belt width } \\
{[\mathrm{m}]}\end{array}$ & $\begin{array}{c}\text { belt speed } \\
{[\mathrm{cm} / \mathrm{s}]}\end{array}$ & $\begin{array}{c}f_{M} \\
{[\text { objects } / \mathrm{s}]}\end{array}$ \\
\hline 1 & 5 & 5.2 & 1 & 10 & 37 \\
2 & $2.5-10$ & 6.45 & 1 & 20 & 48 \\
3 & $2.5-10$ & 6.45 & 2 & 10 & 48 \\
\hline
\end{tabular}

A uniform random distribution determines where the next object is to be released along the width of the belt, while objects are released one by one with a constant time interval. This release strategy minimizes the probability of overlap, meaning that any other distribution or non-uniform time interval will on average result in more overlaps. The differences between mono and size-range feeding are surprisingly small in Fig. 14.4, but still noticeable at low feed rates. A similar conclusion applies to the two options for increasing the feed rate capacity, which are using a wider belt and a higher belt speed. If it is acceptable that at most $10 \%$ of the objects overlap, the feed rate should lie between $8-10 \%$ of the maximum in which case $8-10.5 \%$ of the belt surface will be covered with objects.

But not all overlaps result in a separation error. Overlaps between only targeted or only non-targeted objects may turn out ok. For mixed overlaps the sensor may generate false positives or false negatives, which affect either the purity of the main product or the recovery of the targeted objects. To better quantify the overlap error the simulation may be extended to keep track of the nature of each object and the overlaps. Combined with knowledge of the composition of the feed, the simulation can then predict the 
Table 14.2: Examples of Mechanical Separation Techniques and Attributes.

\begin{tabular}{ll}
\hline Resistance techniques & \multicolumn{1}{c}{ Involved object attributes } \\
\hline $\begin{array}{l}\text { friction } \\
\text { drag }\end{array}$ & $\begin{array}{l}\text { surface conditions; mass } \\
\text { size; shape; Reynolds number } \\
\text { buoyancy } \\
\text { mesh or aperture }\end{array}$ \\
impact & $\begin{array}{l}\text { size; shape } \\
\text { elasticity; mass; shape; damping }\end{array}$ \\
Stimulus techniques & Involved object attributes \\
fluid flow & mass density; drag \\
vibrations & mass density; elasticity; size \\
centrifugal force & mass \\
magnetic field & permeability; size \\
electric field & conductivity; permittivity; size \\
EM-induction & conductivity; magnetization; mass
\end{tabular}

percentage of false positives and negatives due to overlapping objects. Computer simulations can be extended even further to closely match the higher complexity of object dynamics during feeding. For example, it may account for varying object size and shape, randomness in position and alignment where objects can drop on the conveyor belt, and the probability that an object could bounce from the belt or from another object lying on the belt.

\subsubsection{Designing mechanical separation technology}

For mechanical separation, the targeted objects must be forced into a different trajectory than the rest of the feed on the basis of the discerning attributes. To this end, an attribute-specific force has to be applied that selectively obstructs (resistance technique) or accelerates (stimulus technique) the targeted objects. In both categories it may be a concentrated force or a small force exerted over a longer distance. A concentrated force tends to lead to a smaller machine that requires less space. Table 14.2 shows examples of the many possible techniques falling in the two categories and the associated main attributes. Different principles may be combined to form a hybrid technique, in which case more than one unique attribute may be utilized to improve the sensitivity of the separation.

Whichever approach is chosen, three phases may be discerned for the design of a mechanical separator. Phases 1 and 2 provide scientifically founded recommendations, leading to an assembly of the effective prototype in phase 3 . One typically enters phase 1 on the basis of an inspired idea. Such a creative event can be the birth of novel technology, but it has 
no bearing on science other than that it sparks curiosity and stimulates the imagination.

Design phase 1: The physical principles are subjected to analytical assessment to establish the potential separation subspace and sensitivity. Directed physical experiments may be part of this phase to assess if all the main principles are carefully taken into account. Next, the mixed waste streams holding relevant material classes with unique attributes corresponding to the separation subspace may be identified, which requires the market knowledge of an industrial partner. Subsequently, the proposed technique can be judged on its potential for creating added value. This phase provides insights into the technical capabilities and the economic potential of the proposed technique.

Design phase 2: This phase involves more statistics to determine more reliably the optimum separation subspace and to improve the sensitivity. Included are the designs for a method for material feeding and a method for guiding the separated materials into the main product or residue. Attention has to be paid to measures that prevent blocking and minimize damage mechanisms such as fouling, wear and tear. First implementations of the different subsystems may be tested, separately or connected, for their performance.

Design phase 3: The subsystems are integrated into a functional prototype, which is performance tested using prepared batches and, finally, real mixed waste. Analysis of the test data may indicate shortcomings and bottlenecks. These require an in-depth investigation to understand the underlying physics in as much detail as is required for an efficient solution.

After the last design phase the prototype may be transferred from the research environment to the industrial partner, where engineering is usually required to meet special demands. For example, variable machine settings to improve operator control, automated sensor control and material flow monitoring capabilities, modifications to reduce or facilitate maintenance, and compliance with regulations for worker safety, noise and dust.

\subsubsection{Technology starts with a principle}

Seemingly simple physical principles can give rise to a diversity of powerful mechanical separation techniques by changing the conditions in some extreme way. Important examples are the trajectory techniques. There, objects are directed to follow a path during which they are subjected to a 
selective force that significantly modifies the path of the targeted objects to where they can be collected separately. For example, a path in air or water where objects are subjected to drag, or a path that leads objects along a rough surface or a surface equipped with attribute-selective obstacles (e.g. riffles). The mathematical analysis of shaped solid objects following a 3D path generally demands a computer to solve the equations. Fortunately, the physical principles are much easier to explain.

Ballistic separation: An object at some height above the floor is given an initial velocity under an upward angle after which it is left to gravity and drag to determine where the object will drop onto the floor. It is readily found that for equal-sized objects the heavy ones tend to travel farther from the launching point than the light ones. This forms a basis for separating light from heavy objects. Similarly, for objects with the same mass the ones with a small cross section in the direction of motion tend to travel farther than those with a large cross section. This forms a basis for separating objects according to shape (e.g. flat, round). A complication is that drag is influenced by both shape and size, which means both attributes play a role in ballistic separation. In a practical setting the choice of fluid (e.g. water or air) influences the amount of drag and therefore the space needed to house a ballistic separator.

Friction separation: An object is given an initial horizontal velocity at the top of an inclined surface, after which it is left to gravity and surface friction to determine where the object will drop into a collector. It is readily experienced that for equal-sized objects the ones with a low level of friction tend to travel farther from the launching point than the ones with a high level of friction. This forms a basis for separating objects on the basis of surface resistance. For example rubbers can be separated from smooth plastics, or round rolling objects from flat ones.

Sink-float separation: If an object is dropped into a water tank its density in relation to water determines if it eventually sinks or floats, as discovered by Archimedes. The light fraction can be collected by letting the water flow in a horizontal direction and spill over a lowered edge of the tank or by pulling a sieve along the water surface. This is the principle behind the sinkfloat techniques, for which different liquids are available. The throughput capacity depends partly on how fast the objects sink or move back to the liquid surface after being dropped into the tank, which is influenced by object size and drag. 
The concept of sink-float may be extended by using a magnetizable liquid, called ferrous liquid, and applying a gradient magnetic field using either a permanent magnet or an electromagnet. In this configuration the magnetic field gradient produces a gradient in the effective density of the liquid. Objects with a targeted density will concentrate at a certain distance from the magnet surface where the effective density of the liquid matches the target density. Materials may be selectively extracted on the basis of density range by making the liquid flow into carefully positioned open slots or by pulling sieves at selected depths through the liquid. This technique is called magnetic gradient density separation, and allows the production of multiple products with different density ranges in one processing step.

Terminal velocity separation: If we take a water tank and generate a vertical flow from bottom to top the conditions are set for terminal velocity separation. If we drop an object in the tank it is subjected to gravity, buoyancy and the enlarged drag exerted by the flowing water. An object that sinks will eventually reach a maximum velocity called terminal velocity, which is determined in a balance between gravity, buoyancy and drag. This may be compared to a falling raindrop that also reaches a terminal velocity. The terminal velocity depends on the Reynolds number, because water flows differently around an object depending on its shape and velocity. If the density of the object is too small it will get flushed from the top of the tank into the light product. If the density is high enough it will sink to the bottom of the tank where it is collected into the heavy product. By varying the water flow one can set the optimum cut-point between the heavy and light materials. This principle is the basis for techniques like elutriation, where heavy objects are separated from light ones, and where objects with large cross sections are separated from those with small cross sections. Though both shape and density play a role, the advantage is that water can be used as a cheap and available medium to separate much denser objects such as metals.

In a variation on this principle one may replace gravity with a centrifugal force by feeding a drum that is rotated around the vertical central axis. The chosen acceleration and terminal velocity may be smaller or larger than the acceleration of gravity. If it is smaller, the flow speed needed for separation must also be smaller, thereby saving some energy on pumping. If the centrifugal acceleration is larger than the acceleration of gravity, the flow needed for separation must be faster, which allows for a higher throughput capacity. 
Other forced flow techniques: The fact that a flowing fluid can lift or move objects is utilized in a wide variety of techniques. Examples are: air suction (concentrates light and airy materials); air knives (concentrates light and air-resistant objects); fluidized beds (separates effectively as in sink-float, using air or a liquid and a homogenous bed of selected density and size particles); cyclones (separates small, light and airy particles from heavy ones), spirals (using water: separates heavy particles from light ones; in air: separates particles that roll from those with high surface friction), and the water table (separates heavy particles from light ones and/or small particles from large ones using a flow of water over an inclined surface; the surface may also be equipped with riffles as a selective obstruction to small, high density particles that may be separated into an additional product).

\section{References}

1. Eurostat. (n.d.). Eurostat waste databases. Retrieved from http://ec.europa .eu/eurostat/web/waste

2. Meadows, D.H., Meadows, D.L., Randers, J., and Behrens, W.W. III. (1972). The limits to growth: A report for The Club of Rome's project on the predicament of mankind. Washington D.C., USA: Universe Books.

3. European Commission. (2008). The Waste Framework Directive 2008/98/EC. Retrieved from http://ec.europa.eu/environment/waste/framework/.

4. Van Der Valk, H.J.L., Braam, B.C., and Dalmijn, W.L. (1986). Eddy-current separation by permanent magnets part I: Theory. Resources and Conservation, 12(3-4), 233-252.

5. Scott, D.M. (1995). A two-colour near-infrared sensor for sorting recycled plastic waste. Measurement Science and Technology, 6(2), 156-159.

6. Ellen MacArthur Foundation. (2013). Towards a circular economy. Cowes, UK: Ellen MacArthur Foundation.

7. Rousta, K., Bolton, K., Lundin, M., and Dahlén, L. (2015). Quantitative assessment of distance to collection point and improved sorting information on source separation of household waste. Waste Management, 40, 22-30.

8. Rousta, K., Ordoñez, I., Bolton, K., and Dahlén, L. (2017). Support for designing waste sorting systems: A mini review. Waste Management and Research, 35(11), 1099-1111.

9. Rahman, A. and Bakker, M.C.M. (2012). Hybrid sensor for metal grade measurement of a falling stream of solid waste particles. Waste Management, 32, $1316-1323$.

10. Isakov, V. (1993). Uniqueness and stability in multi-dimensional inverse problems. Inverse Problems, 9(6), 579-621.

11. Jain, A.K., Murty, M.N., and Flynn, P.J. (1999). Data clustering: A review. ACM Computing Surveys, 31(3), 264-323.

12. Xia H. and Bakker, M.C.M. (2014). Reliable classification of moving waste materials with LIBS in concrete recycling. Talanta, 120, 239-247. 